\title{
The minor C-allele of rs2014355 in ACADS is associated with reduced insulin release following an oral glucose load
}

\author{
Malene Hornbak ${ }^{1,2 \dagger}$, Karina Banasik ${ }^{1 *}{ }^{*}$, Johanne M Justesen ${ }^{1}$, Nikolaj T Krarup ${ }^{3}$, Camilla H Sandholt ${ }^{1,5}$, \\ Åsa Andersson ${ }^{2}$, Annelli Sandbæk ${ }^{6}$, Torsten Lauritzen ${ }^{6}$, Charlotta Pisinger ${ }^{7,9}$, Daniel R Witte ${ }^{8}$, \\ Thorkild IA Sørensen ${ }^{4,9}$, Oluf Pedersen ${ }^{1,3,4,10}$, Torben Hansen ${ }^{1,3,11}$
}

\begin{abstract}
Background: A genome-wide association study (GWAS) using metabolite concentrations as proxies for enzymatic activity, suggested that two variants: rs2014355 in the gene encoding short-chain acyl-coenzyme A dehydrogenase (ACADS) and rs11161510 in the gene encoding medium-chain acyl-coenzyme A dehydrogenase (ACADM) impair fatty acid $\beta$-oxidation. Chronic exposure to fatty acids due to an impaired $\beta$-oxidation may down-regulate the glucose-stimulated insulin release and result in an increased risk of type 2 diabetes (T2D). We aimed to investigate whether the two variants associate with altered insulin release following an oral glucose load or with T2D.

Methods: The variants were genotyped using KASPar ${ }^{\circledR}$ PCR SNP genotyping system and investigated for associations with estimates of insulin release and insulin sensitivity following an oral glucose tolerance test (OGTT) in a random sample of middle-aged Danish individuals $\left(n_{\text {ACADS }}=4,324 ; n_{\text {ACADM }}=4,337\right)$. The T2D-case-control study involved a total of $\sim 8,300$ Danish individuals $\left(n_{\text {ACADS }}=8,313 ; n_{\text {ACADM }}=8,344\right)$.

Results: In glucose-tolerant individuals the minor C-allele of rs2014355 of ACADS associated with reduced measures of serum insulin at 30 min following an oral glucose load (per allele effect $(\beta)=-3.8 \%(-6.3 \% ;-1.3 \%), P=$ $0.003)$, reduced incremental area under the insulin curve $(\beta=-3.6 \%(-6.3 \% ;-0.9 \%), P=0.009)$, reduced acute insulin response $(\beta=-2.2 \%(-4.2 \% ; 0.2 \%), P=0.03)$, and with increased insulin sensitivity $|S|_{\text {Matsuda }}(\beta=2.9 \%(0.5 \% ; 5.2 \%), P$ $=0.02$ ). The $C$-allele did not associate with two other measures of insulin sensitivity or with a derived disposition index. The C-allele was not associated with T2D in the case-control analysis (OR 1.07, 95\% Cl 0.96-1.18, $P=0.21$ ). rs11161510 of ACADM did not associate with any indices of glucose-stimulated insulin release or with T2D.

Conclusions: In glucose-tolerant individuals the minor C-allele of rs2014355 of ACADS was associated with reduced measures of glucose-stimulated insulin release during an OGTT, a finding which in part may be mediated through an impaired $\beta$-oxidation of fatty acids.
\end{abstract}

\section{Background}

Acute exposure of free fatty acids (FFA) to the pancreatic $\beta$-cells is known to both stimulate insulin secretion and modulate the glucose-stimulated insulin secretion (GSIS) whereas chronic exposure leads to desensitization of the insulin receptors and decreased function of the pancreatic $\beta$-cells. Also, chronic exposure to FFA is

\footnotetext{
* Correspondence: karina.banasik@sund.ku.dk

+ Contributed equally

${ }^{1}$ Marie Krogh Center for Metabolic Research, Section of Metabolic Genetics,

Faculty of Health Sciences, University of Copenhagen, Denmark

Full list of author information is available at the end of the article
}

associated with insulin resistance and increased risk of developing diabetes mellitus [1-9].

Recently, Gieger et al. (2008) conducted a genomewide association study (GWAS) in 284 healthy men characterized by quantitative measurements of 363 metabolites obtained from fasting serum samples, and used concentration ratios for the metabolites as proxies for enzymatic activity [10]. They identified with genome-wide significance variants in two genes, one encoding short-chain acyl-coenzyme A dehydrogenase $(A C A D S)$ which is expressed in mitochondria and target

\section{Biomed Central}


short chain fatty acid (SCFA), and one encoding medium-chain acyl-coenzyme A dehydrogenase (ACADM) that target medium chain C4-C12 fatty acids (MCFA), where the corresponding metabolic phenotype matched the biochemical pathway of the enzymes. A strong association $\left(P=9.3 \times 10^{-17}\right)$ was found between rs2014355 in $A C A D S$ and the ratio between the short-chain acylcarnitines C3 and C4. Similarly, they found a strong association $\left(P=7.6 \times 10^{-17}\right)$ between $\mathrm{rs} 11161510$ in $A C A D M$ and the ratio between medium-chain acylcarnitines $\mathrm{C} 8$ and $\mathrm{C} 12$ [10]. These findings indicate that individuals homozygous for the minor alleles of the variants in $A C A D S$ and $A C A D M$ have reduced dehydrogenase activity and therefore are likely to show impaired $\beta$ oxidation. For instance, in situations of prolonged starvation, these individuals may become hypoglycemic and show hypoglycemia-related symptoms [10].

In pancreatic $\beta$-cells, glucose is the primary source of fuel to stimulate insulin secretion $[1,2,9]$. However, when the glucose concentration in plasma is low, fatty acids (FA) are produced by de novo lipolysis of endogenous triglycerides (TG) increasing the level of cytoplasmic long-chain Coenzyme A molecules. Subsequently, these molecules are transported into the mitochondria (via carnitine-palmitoyl-transferase I (CPT-I)) for $\beta$-oxidation leading to increased cellular ATP levels. Thus, the cell can use FAs as fuels to produce both energy and stimulate the insulin secretion [8]. Conversely, when plasma glucose concentration is elevated, $\beta$-oxidation of FAs is inhibited due to allosteric inhibition of CPT-I from malonyl-CoA with a subsequent increase in FFA esterification and synthesis of complex lipid molecules such as diacylglycerol, triacylglycerol and fatty acids $[1,7,8,11,12]$. Accordingly, during chronically elevated circulating FFA levels it is hypothesized that the synergism of both glucose- and lipid-stimulated insulinsecretion elevates the basal insulin secretion causing hyperinsulinemia which may eventually exhaust the $\beta$-cell and desensitize the insulin receptors [5]. Chronic FA exposure may thus down-regulate the GSIS with overt diabetes as a consequence [5].

$A C A D S$ and $A C A D M$ encode Acyl-CoA dehydrogenases, which are enzymes that catalyze the initial step in the $\beta$-oxidation of FAs in the mitochondria, each targeting FAs of varying length. In humans, deficiency of these enzymes leads to low tissue levels of dehydrogenase activity and improper break-down of SCFA and MCFA. Consequently, the FAs cannot be converted into energy in situations where the glucose-concentration is low resulting in hypoglycemia, lethargy, hypotonia, and seizures [13-15]. ACADS is very abundant in the pancreatic $\beta$-cell, indicating that it plays an important regulatory function, and decreased activity of short-chain dehydrogenases in the pancreatic $\beta$-cell is hypothesized to impair insulin secretion [15]. Studies in mice deficient for ACADS and ACADM showed that the mice developed fatty liver, changes in hepatic carbohydrate metabolism, and hypoglycemia when fasted, and studies by Herrema et al. indicated that peripheral rather than hepatic consequences might underlie the hypoglycemia associated with disorders of mitochondrial fatty acid oxidation [16-19].

The aim of the present study was to investigate rs 2014355 of $A C A D S$ and rs 11161510 of $A C A D M$ in relation to indices of insulin release, insulin sensitivity, and fasting serum lipid levels in a large random sample of middle-aged individuals $(n=6,162)$; also we examined the putative relation to T2D prevalence in a casecontrol study involving $\sim 8,300$ Danish individuals $\left(n_{A C A D S}=8,313 ; n_{A C A D M}=8,344\right)$.

\section{Methods}

\section{Study population}

The study population included 10,276 Danes from four different study groups: 1) The Inter99 cohort which is a randomized, non-pharmacological intervention study for the prevention of ischemic heart disease in people recruited as a random sample and conducted at the research Centre for Prevention and Health in Glostrup, Copenhagen $(n=6,162)$ (ClinicalTrials.gov ID-no: NCT00289237); 2) T2D patients sampled through the out-patient clinic at Steno Diabetes Center (SDC) $(n=$ $1,695) ; 3)$ a randomly recruited sample of middle-aged glucose-tolerant participants examined at SDC $(n=$ 810); and 4) T2D patients from the Addition Denmark screening study cohort (Anglo-Danish-Dutch Study of Intensive Treatment in People with Screen-Detected Diabetes in Primary Care) (ClinicalTrials.gov ID-no: NCT00237548), which is a population-based, high-risk screening and intervention study for T2D in general practice $(n=1,609)[20,21]$. Characteristics for individuals included in the study are shown in Additional file 1. All participants in study group 1 and 3 underwent a standard $75 \mathrm{~g}$ oral glucose tolerance test (OGTT). T2D and glucose tolerance status were diagnosed according to the World Health Organization 1999 criteria [22].

Analyses of quantitative diabetes-related traits were performed in glucose-tolerant individuals from the Inter99 cohort $\left(n_{A C A D S}=4,324 ; n_{A C A D M}=4,337\right)$. Analyses of fasting serum lipid levels were performed in glucose-tolerant and treatment-naïve individuals from the Inter99 cohort $\left(n_{A C A D S}=5,702 ; n_{A C A D M}=5,731\right)$. Study group 3 was excluded from the quantitative trait analyses due to missing phenotypes. All T2D patients and glucose-tolerant individuals were included in the casecontrol study of T2D $\left(n_{A C A D S}=8,313 ; n_{A C A D M}=8,344\right)$. Participants were of Danish nationality and informed written consent was obtained from all individuals before 
participation. The studies were conducted in accordance with the Declaration of Helsinki II and were approved by the local Ethical Committees of Copenhagen and Aarhus.

\section{Biochemical and anthropometric measures}

Height (without shoes) and weight were measured in light indoor clothing, and BMI was calculated as weight $(\mathrm{kg}) /$ height $^{2}\left(\mathrm{~m}^{2}\right)$. Waist circumference was measured in the upright position midway between the iliac crest and the lower costal margin, and hip circumference was measured at its maximum [21]. Blood samples were drawn after a $12 \mathrm{~h}$ overnight fast. Plasma glucose was analyzed by a glucose oxidase method (Granutest; Merck, Darmstadt, Germany) and serum insulin (excluding des-31,32 and intact proinsulin) was measured using the Autodelfia insulin kit (Perkin-Elmer/ Wallac, Turku, Finland). Serum triacylglycerol was analyzed using enzymatic colorimetric methods (GPO-PAP and CHOD-PAP; Roche Molecular Biochemicals, Mannheim, Germany). Serum C-peptide concentrations were measured by a time-resolved fluoroimmunoassay (AutoDELFIA C-peptide kit; Perkin-Elmer/Wallac, Turku, Finland). Homeostasis model assessment of insulin resistance (HOMA-IR) was calculated as: (fasting plasma glucose $(\mathrm{mmol} / \mathrm{l}) \times$ fasting serum insulin $(\mathrm{pmol} / \mathrm{l})) / 22.5$ [23]. BIGTT-insulin sensitivity index $\left(\right.$ BIGTT- $\left.\mathrm{S}_{\mathrm{i}}\right)$ and BIGTT-acute insulin response (BIGTT-AIR) use information on sex and BMI combined with analysis of plasma glucose and serum insulin levels at time points 0,30 , and $120 \mathrm{~min}$ to provide indices for $S_{\text {I }}$ and AIR that highly correlate with indices obtained during an intravenous glucose tolerance test. These indices were calculated as described elsewhere [24]. $\beta$-cell function was further estimated as the insulinogenic index, calculated as: (serum insulin at $30 \mathrm{~min}(\mathrm{pmol} / \mathrm{l})$ - fasting serum insulin $(\mathrm{pmol} / \mathrm{l})) /($ plasma glucose at $30 \mathrm{~min}$ $(\mathrm{mmol} / \mathrm{l})$ - fasting plasma glucose $(\mathrm{mmol} / \mathrm{l}))$. A disposition index was calculated as insulinogenic index/ HOMA-IR. Matsuda whole body insulin sensitivity index $\left(\mathrm{ISI}_{\text {Matsuda }}\right)$ was calculated as $(10,000 / \sqrt{ }$ (fasting plasma glucose $\times$ fasting serum insulin $) \times($ mean plasma glucose $\times$ mean serum insulin during OGTT)) [25]. Area under the curve (AUC) for plasma glucose, serum insulin and serum $\mathrm{C}$-peptide were calculated using the trapezoidal method. The AUC for insulin/AUC for glucose ratio was calculated as AUC for insulin/AUC for glucose.

\section{Genotyping}

Genotyping was performed using KASPar ${ }^{\circledR}$ PCR SNP genotyping system (KBiosciences, Herts, UK) with a success rate $>97 \%$. Discordance was $<0.1 \%$ as judged from re-genotyping of 1187 random duplicate samples. Allele frequencies were in accordance with HapMap (CEU population) and the study by Gieger et al., and obeyed Hardy-Weinberg equilibrium $(P>0.05)$ [10].

\section{Statistical analysis}

A general linear model was applied for testing quantitative traits in relation to genotype. Non-normally distributed data (measures of serum insulin, serum triglyceride, and serum C-peptide levels, HOMA-IR, insulinogenic index, AUC for insulin/AUC for glucose ratio, ISI $_{\text {Matsuda, and BIGTT-AIR) were logarithmically }}$ transformed before analyses. Logistic regression was used to examine differences in genotype distribution in the case-control studies. All studies were performed in R 2.10.0 assuming an additive model and adjusted for age, sex, and BMI. In a subset of the analyses, estimates of $\beta$-cell function were additionally adjusted for fasting serum lipid levels (cholesterol or triglyceride). $P<0.05$ were considered significant. The statistical power calculations were done using CaTS, power calculations for large genetic association studies, available at http://www.sph. umich.edu/csg/abecasis/cats/. The statistical power to detect an OR of 1.10 was estimated to be $80 \%$ for rs2014355 of $A C A D S$ and $86 \%$ for rs11161510 of $A C A D M$, respectively. $(P<0.05, \mathrm{MAF}=24 \%, \mathrm{~T} 2 \mathrm{D}$ risk $=0.08)$.

\section{Results}

\section{Association with T2D-related quantitative traits}

The impact of $A C A D S$ rs2014355 and ACADM rs11161510 on OGTT-derived diabetes-related quantitative traits was evaluated in a random sample of glucosetolerant individuals from the Inter99 cohort (Table 1 and 2). Assuming an additive genetic model, carriers of the minor C-allele of ACADS rs2014355 had decreased measures of serum insulin (per allele effect $(\beta)=-3.8 \%$ $(-6.3 \% ;-1.3 \%), P=0.003)$ at $30 \mathrm{~min}$, and decreased incremental AUC for serum insulin from 0-120 minutes during an OGTT $(\beta=-3.6 \%(-6.3 \% ;-0.9 \%), P=0.009)$ (Table 1). Also, measures of serum $C$-peptide at $30 \mathrm{~min}$ $(\beta=-1.8 \%(-3.4 \% ;-0.1 \%), P=0.03)$, and incremental AUC for serum $C$-peptide from $0-120$ minutes $(\beta=$ $-1.8 \%(-3.4 \% ;-0.1 \%), P=0.03)$ during an OGTT were decreased for carriers of the rs2014355 C-allele (Table 1). Additionally, the rs $2014355 \mathrm{C}$-allele of ACADS associated with reduced $\beta$-cell response measured as BIGTT-AIR ( $\beta=-2.2 \%(-4.2 \% ; 0.2 \%), P=0.03)$ and the AUC for insulin/AUC for glucose ratio $(\beta=-0.03 \%$ (-0.05\%;-0.01\%), $P=0.01)$. The $\mathrm{C}$-allele did not associate with BIGTT-S $S_{i}$ or HOMA-IR or with a HOMA-IRderived disposition index; however, the variant associated with increased insulin sensitivity according to $\operatorname{ISI}_{\text {Matsuda }}(\beta=2.9 \%(0.5 \% ; 5.2 \%), P=0.02)$ (Table 1$)$. When measures of insulin release were adjusted for either fasting levels of serum cholesterol or triglyceride, 
Table 1 Anthropometric and metabolic characteristics of successfully genotyped glucose-tolerant Danes from the Inter99 cohort stratified according to ACADS rs2014355 (T/C) and ACADM rs11161510 (C/T) genotype, respectively

\begin{tabular}{|c|c|c|c|c|c|c|}
\hline & & WT & $\mathrm{HE}$ & $\mathrm{HO}$ & $\beta(95 \% \mathrm{Cl})$ & $P_{\text {add }}$ \\
\hline \multirow{2}{*}{$\begin{array}{l}n \text { (men/ } \\
\text { women) }\end{array}$} & ACADS & $2,576(1,208 / 1,368)$ & $1,496(694 / 802)$ & $252(105 / 147)$ & & \\
\hline & ACADM & $2,121(991 / 1,130)$ & $1,821(825 / 996)$ & 395(186/209) & & \\
\hline \multirow[t]{2}{*}{ Age (years) } & & $45 \pm 8$ & $45 \pm 8$ & $45 \pm 8$ & & \\
\hline & & $45 \pm 8$ & $45 \pm 8$ & $46 \pm 8$ & & \\
\hline \multirow[t]{2}{*}{ BMI $\left(\mathrm{kg} / \mathrm{m}^{2}\right)$} & & $25.5 \pm 4.1$ & $25.4 \pm 3.9$ & $25.8 \pm 4.0$ & $0.03(-0.16 ; 0.23)$ & 0.74 \\
\hline & & $25.5 \pm 4.0$ & $25.6 \pm 4.2$ & $25.4 \pm 3.8$ & $0.03(-0.16 ; 0.21)$ & 0.79 \\
\hline \multicolumn{7}{|c|}{ Plasma glucose (mmol/l) } \\
\hline \multirow[t]{2}{*}{ Fasting } & ACADS & $5.3 \pm 0.4$ & $5.3 \pm 0.4$ & $5.3 \pm 0.4$ & $0.005(-0.013 ; 0.024)$ & 0.55 \\
\hline & ACADM & $5.3 \pm 0.4$ & $5.3 \pm 0.4$ & $5.3 \pm 0.4$ & $0.016(-0.001 ; 0.033)$ & 0.06 \\
\hline $\begin{array}{l}\text { 30-min } \\
\text { during }\end{array}$ & & $8.2 \pm 1.5$ & $8.2 \pm 1.5$ & $8.1 \pm 1.6$ & $-0.05(-0.12 ; 0.02)$ & 0.18 \\
\hline an OGTT & & $8.2 \pm 1.5$ & $8.1 \pm 1.5$ & $8.3 \pm 1.5$ & $-0.02(-0.08 ; 0.05)$ & 0.60 \\
\hline $\begin{array}{l}\text { 120-min } \\
\text { during }\end{array}$ & & $5.5 \pm 1.1$ & $5.5 \pm 1.2$ & $5.5 \pm 1.1$ & $-0.03(-0.08 ; 0.03)$ & 0.36 \\
\hline an OGTT & & $5.5 \pm 1.1$ & $5.5 \pm 1.1$ & $5.5 \pm 1.2$ & $-0.004(-0.05 ; 0.05)$ & 0.88 \\
\hline Incremental & & $183.7 \pm 100.3$ & $177.9 \pm 102.0$ & $175.5 \pm 100.7$ & $-4.90(-9.82 ; 0.01)$ & 0.05 \\
\hline $\mathrm{AUC}$ & & $183.7 \pm 99.3$ & $177.5 \pm 100.8$ & $183.3 \pm 103.7$ & $-2.9(-7.5 ; 1.6)$ & 0.21 \\
\hline \multicolumn{7}{|c|}{ Serum insulin (pmol/l) } \\
\hline \multirow[t]{2}{*}{ Fasting } & ACADS & $31(22-46)$ & $31(22-46)$ & $29(23-41)$ & $-1,9 \%(-4.3 \% ; 0.6 \%)$ & 0.14 \\
\hline & ACADM & $31(22-46)$ & $31(22-45)$ & $31(23-46)$ & $0.3 \%(-2.0 \% ; 2.6 \%)$ & 0.83 \\
\hline $\begin{array}{l}\text { 30-min } \\
\text { during }\end{array}$ & & $246(177-356)$ & $239(175-329)$ & $234(181-302)$ & $-3.8 \%(-6.3 \% ;-1.3 \%)$ & 0.003 \\
\hline an OGTT & & $239(175-337)$ & $244(178-351)$ & $245(173-348)$ & $0.5 \%(-1.8 \% ; 2.8 \%)$ & 0.65 \\
\hline $\begin{array}{l}\text { 120-min } \\
\text { during }\end{array}$ & & $137(88-212)$ & $139(86-209)$ & $130(87-189)$ & $-1.1 \%(-4.5 \% ; 2.4 \%)$ & 0.51 \\
\hline an OGTT & & $140(87-212)$ & $136(88-208)$ & $129(80-214)$ & $-2.8 \%(-5.9 \% ; 0.3 \%)$ & 0.08 \\
\hline Incremental & & $18,220(12,600-25,830)$ & $17,260(12,700-23,980)$ & $\begin{array}{c}16,200(12,630- \\
22,720)\end{array}$ & $-3.6 \%(-6.3 \% ;-0.9 \%)$ & 0.009 \\
\hline AUC & & $17,740(12,720-24,780)$ & $17,780(12,600-25,380)$ & $\begin{array}{c}17,670(12,490- \\
25,790)\end{array}$ & $0.1 \%(-2.3 \% ; 2.6 \%)$ & 0.91 \\
\hline \multicolumn{7}{|c|}{ Serum C-peptide (pmol/l) } \\
\hline \multirow[t]{2}{*}{ Fasting } & ACADS & $495(392-632)$ & $499(394-642)$ & $484(399-627)$ & $0.2 \%(-1.3 \% ; 1.8 \%)$ & 0.78 \\
\hline & ACADM & $498(393-639)$ & $494(392-632)$ & $496(405-627)$ & $-0.4 \%(-1.8 \% ; 1.0 \%)$ & 0.61 \\
\hline $\begin{array}{l}\text { 30-min } \\
\text { during }\end{array}$ & & $1,910(1,490-2,390)$ & $1,840(1,470-2,310)$ & $1,820(1,450-2,268)$ & $-1.8 \%(-3.4 \% ;-0.1 \%)$ & 0.03 \\
\hline an OGTT & & $1,880(1,480-2,350)$ & $1,870(1,490-2,398)$ & $1,870(1,450-2,295)$ & $0.3 \%(-1.2 \% ; 1.8 \%)$ & 0.71 \\
\hline $\begin{array}{l}\text { 120-min } \\
\text { during }\end{array}$ & & $1,960(1,500-2,490)$ & $1,980(1,490-2,490)$ & $1,940(1,450-2,410)$ & $-0.2 \%(-2.0 \% ; 1.7 \%)$ & 0.84 \\
\hline an OGTT & & $1,980(1,500-2,508)$ & $1,960(1,490-2,480)$ & $1,960(1,480-2,490)$ & $-1.3 \%(-3.0 \% ; 0.4 \%)$ & 0.13 \\
\hline Incremental & & $\begin{array}{c}149,800(117,600- \\
185,000)\end{array}$ & $\begin{array}{c}147,300(117,700- \\
181,600)\end{array}$ & $\begin{array}{c}138,500(117,400- \\
178,400)\end{array}$ & $-1.8 \%(-3.4 \% ;-0.1 \%)$ & 0.03 \\
\hline AUC & & $\begin{array}{c}148,800(117,100- \\
184,100)\end{array}$ & $\begin{array}{c}148,000(118,500- \\
183,200)\end{array}$ & $\begin{array}{c}147,900(116,600- \\
183,800)\end{array}$ & $-0.1 \%(.1 .7 \% ; 1.4 \%)$ & 0.85 \\
\hline \multicolumn{7}{|c|}{ Derived indices } \\
\hline HOMA-IR & ACADS & $7.5(5.2-11.0)$ & $7.4(5.1-11.0)$ & $6.8(5.3-9.6)$ & $-1.8 \%(-4.4 \% ; 0.7 \%)$ & 0.16 \\
\hline $\begin{array}{l}(\mathrm{mmol} / \\
\text { |**mol/l) }\end{array}$ & ACADM & $7.4(5.1-11.0)$ & $7.5(5.2-10.9)$ & $7.4(5.3-11.0)$ & $0.6 \%(-1.8 \% ; 3.0 \%)$ & 0.63 \\
\hline \multirow[t]{2}{*}{$\overline{B I G T T-S_{i}}$} & & $10.3 \pm 3.7$ & $10.4 \pm 3.8$ & $10.6 \pm 3.5$ & $0.14(-0.05 ; 0.34)$ & 0.14 \\
\hline & & $10.4 \pm 3.7$ & $10.3 \pm 3.7$ & $10.3 \pm 3.6$ & $-0.01(-0.19 ; 0.17)$ & 0.93 \\
\hline Insulinogenic & & $79.9(48.9-130.7)$ & 74.7 (49.2-123.4) & $77.1(51.5-121.7)$ & $-0.8 \%(4.2 \% ; 2.5 \%)$ & 0.63 \\
\hline index & & $75.9(48.2-123.9)$ & $80.0(51.1-136.8)$ & $75.8(46.5-126.4)$ & $2.6 \%(0.5 \% ; 5.7 \%)$ & 0.10 \\
\hline
\end{tabular}


Table 1 Anthropometric and metabolic characteristics of successfully genotyped glucose-tolerant Danes from the Inter99 cohort stratified according to ACADS rs2014355 (T/C) and ACADM rs11161510 (C/T) genotype, respectively (Continued)

\begin{tabular}{|c|c|c|c|c|c|}
\hline Disposition & $9.3(5.1-17.7)$ & $9.3(5.1-18.1)$ & $10.6(5.7-18.7)$ & $0.7 \%(-3.1 \% ; 4.5 \%)$ & 0.73 \\
\hline index & $9.4(5.3-18.2)$ & $9.4(5.0-17.6)$ & $9.2(4.9-16.7)$ & $-3.5 \%(7.9 \% ; 0.8 \%)$ & 0.11 \\
\hline \multirow[t]{2}{*}{$|S|_{\text {Matsuda }}$} & $25.2(17.8-35.4)$ & $26.1(18.2-35.9)$ & $27.9(20.1-36.0)$ & $2.9 \%(0.5 \% ; 5.2 \%)$ & 0.02 \\
\hline & $26.0(18.0-35.4)$ & $25.3(17.9-35.7)$ & $25.6(18.1-35.7)$ & $-0.2 \%(2.3 \% ; 2.0 \%)$ & 0.87 \\
\hline \multirow[t]{2}{*}{ BIGTT-AIR } & $1,690(1,344-2,149)$ & $1,655(1,356-2,056)$ & $1,625(1,317-2,067)$ & $-2.2 \%(-4.2 \% ; 0.2 \%)$ & 0.03 \\
\hline & $1,668(1,356-2,099)$ & $1,684(1,351-2,140)$ & $1,712(1,303-2,048)$ & $0.2 \%(-1.6 \% ; 2.0 \%)$ & 0.83 \\
\hline AUCinsulin/ & $27.8(20.2-38.4)$ & $27.0(20.5-36.1)$ & $24.9(19.8-35.1)$ & $\begin{array}{c}-0.03 \%(-0.05 \% ;- \\
0.01 \%)\end{array}$ & 0.01 \\
\hline AUCglucose & $27.1(20.3-37.0)$ & $27.6(20.4-38.2)$ & $27.7(19.8-38.3)$ & $\begin{array}{c}0.002 \%(-0.019 \% \\
0.023 \%)\end{array}$ & 0.87 \\
\hline
\end{tabular}

\begin{tabular}{|c|c|c|c|c|c|c|c|c|}
\hline \multicolumn{9}{|c|}{ Genotype distribution and allele frequency among patients with T2D and glucose-tolerant control individuals } \\
\hline & & $n$ (men/women) & WT (\%) & HE (\%) & $\mathrm{HO}(\%)$ & $\begin{array}{c}\text { MAF } \\
(95 \% \mathrm{Cl})\end{array}$ & $\mathrm{OR}(95 \% \mathrm{Cl})$ & $P_{\text {add }}$ \\
\hline ACADS & NGT & $4,824(2,239 / 2,585)$ & $2,872(59.5)$ & $1,673(34.7)$ & $279(5.8)$ & $\begin{array}{c}23.1(22.3- \\
24.0)\end{array}$ & $\begin{array}{c}1.07(0.96- \\
1.18)\end{array}$ & 0.21 \\
\hline rs2014355 & $\mathrm{T} 2 \mathrm{D}$ & $3,489(2,063 / 1,426)$ & $2,030(58.2)$ & 1,243 (35.6) & $216(6.2)$ & $\begin{array}{c}24.0(23.0- \\
25.0)\end{array}$ & & \\
\hline ACADM & NGT & $4,841 \quad(2,237 / 2,604)$ & 2,353 (48.6) & $2,053(42.4)$ & $435(9.0)$ & $\begin{array}{c}30.2(29.3- \\
31.1)\end{array}$ & $\begin{array}{c}1.01(0.92- \\
1.11)\end{array}$ & 0.80 \\
\hline rs11161510 & $\mathrm{T} 2 \mathrm{D}$ & $3,503(2,083 / 1,420)$ & $1,686(48.1)$ & $1,486(42.4)$ & $331(9.4)$ & $\begin{array}{c}30.7(29.6- \\
31.8)\end{array}$ & & \\
\hline
\end{tabular}

Data are unadjusted means \pm SD or medians (interquartile range). Values of serum insulin/C-peptide and values derived from serum insulin variables were logarithmically transformed before statistical analysis, and their effect sizes are presented as the increase/decrease in percent. All analyses were made using an additive genetic model, adjusting for age, sex, and BMI. The bottom part of the table include number of individuals divided into genotype groups (\% in each group), and frequencies of the minor allele (MAF) in percentages. Logistic regression was used to compare allele frequencies $\left(P_{\text {add }}\right)$. The odds ratios $(\mathrm{OR})$ and the $95 \%$ confidence interval $(\mathrm{Cl})$ are given for comparison of allele frequency. NGT: Glucose-tolerant individuals, T2D: type 2 diabetic patients. ACADS rs2014355 genotypes: WT, homozygous major T-allele carriers; HE, heterozygous; HO, homozygous minor C-allele carriers. ACADM rs11161510 genotypes: WT, homozygous major C-allele carriers; $\mathrm{HE}$, heterozygous; $\mathrm{HO}$, homozygous minor T-allele carriers.

the effects were unchanged (data not shown). However, adjusting $\mathrm{ISI}_{\text {Matsuda }}$ for serum cholesterol or triglyceride levels strengthened the association with whole body insulin sensitivity ( $P=0.01$, data not shown). Further, when investigating whether rs2014355 influences lipid characteristics in normoglycemic and hyperglycemic individuals, respectively, no effect was evident among normoglycemic individuals (Table 2). However, hyperglycemic (IFG, IGT, and screen-detected T2D) individuals showed a significant genotype-dependent decrease in fasting serum HDL-cholesterol levels $(\beta=-0.036$ (-0.067; -0.005), $P=0.02)$ and increased fasting serum triglyceride levels $(\beta=5.2 \%(0.4 \% ; 10.0 \%), P=0.02)$ among homozygous minor C-allele carriers (Table 2). No significant associations were observed in relation to levels of plasma glucose for this variant (Table 1).

When investigated in all 5,702 treatment-naïve individuals (NGT, IFG, IGT, and screen-detected diabetics) the C-allele of rs2014355 associated with reduced serum insulin levels 30 minutes after an oral glucose load $(\beta=$ $-2.8 \%(-5.0 \% ;-0.5 \%), P=0.02)$, and reduced AUC for serum insulin of $(\beta=-3.2 \%(-5.7 \% ;-0.7 \%), P=0.01)$. The $\mathrm{C}$-allele associated with increased whole body insulin sensitivity estimated according to ISI $_{\text {Matsuda }}$ ( $\beta=2.4 \%(0.3 \% ; 4.6 \%), P=0.03)$ but not with BIGTT-S $\mathrm{S}_{\mathrm{i}}$, HOMA-IR, or disposition index (data not shown). We observed no significant associations between rs11161510 of $A C A D M$ and any of the investigated quantitative diabetes-related traits (Table 1 ).

\section{Case-control analysis of T2D}

Case-control studies of rs2014355 of ACADS and rs11161510 of $A C A D M$ were performed in relation to T2D (Table 1); however, none of the variants were significantly associated with the risk of T2D.

The variants were also tested for association with traits related to obesity (BMI, waist, and waist-hip ratio) in both glucose-tolerant (Additional file 2) and in all treatmentnaïve individuals; however, no associations were found.

\section{Discussion}

In the present study, we investigated rs2014355 of $A C A D S$ and rs11161510 of $A C A D M$ in relation to indices of insulin release and insulin sensitivity as well as their putative relation to the prevalence of T2D. In glucose-tolerant individuals from the Inter99 cohort 
Table 2 Fasting serum lipid levels of successfully genotyped normoglycemic and hyperglycemic Danes from the Inter99 cohort stratified according to ACADS rs2014355 genotype

\begin{tabular}{|c|c|c|c|c|c|}
\hline & WT & $\mathrm{HE}$ & $\mathrm{HO}$ & $\beta(95 \% \mathrm{Cl})$ & $P_{\text {add }}$ \\
\hline \multicolumn{6}{|l|}{ Normoglycemic } \\
\hline$n$ (men/women) & 2576(1208/1368) & 1496(694/802) & 252(105/147) & & \\
\hline Age (years) & $45.2 \pm 7.8$ & $45.4 \pm 7.9$ & $44.9 \pm 7.9$ & & \\
\hline \multicolumn{6}{|c|}{ Lipid characteristics (mmol/l) } \\
\hline Total serum cholesterol & $5.4 \pm 1.0$ & $5.4 \pm 1.0$ & $5.4 \pm 1.1$ & $0.001(-0.046 ; 0.048)$ & 0.97 \\
\hline HDL-cholesterol & $1.5 \pm 0.4$ & $1.5 \pm 0.4$ & $1.5 \pm 0.4$ & $0.002(-0.016 ; 0.019)$ & 0.86 \\
\hline LDL-cholesterol & $3.5 \pm 1.0$ & $3.5 \pm 1.0$ & $3.5 \pm 1.0$ & $-0.001(-0.065 ; 0.063)$ & 0.97 \\
\hline VLDL-cholesterol & $0.5 \pm 0.3$ & $0.6 \pm 0.3$ & $0.5 \pm 0.2$ & $0.007(-0.012 ; 0.026)$ & 0.46 \\
\hline Serum triglyceride & $1.0(0.7-1.4)$ & $1.0(0.7-1.4)$ & $1.0(0.7-1.3)$ & $0.5 \%(-1.8 \% ; 2.7 \%)$ & 0.69 \\
\hline \multicolumn{6}{|l|}{ Hyperglycemic } \\
\hline$n$ (men/women) & $804(473 / 331)$ & 496(306/190) & $78(49 / 29)$ & & \\
\hline Age (years) & $48.8 \pm 7.4$ & $49.4 \pm 7$ & $47.8 \pm 8.1$ & & \\
\hline \multicolumn{6}{|c|}{ Lipid characteristics (mmol/l) } \\
\hline Total serum cholesterol & $5.8 \pm 1.1$ & $5.9 \pm 1.2$ & $5.9 \pm 1.2$ & $0.09(-0.01 ; 0.19)$ & 0.08 \\
\hline HDL-cholesterol & $1.4 \pm 0.4$ & $1.3 \pm 0.4$ & $1.3 \pm 0.5$ & $-0.036(-0.067 ;-0.005)$ & 0.02 \\
\hline LDL-cholesterol & $3.7 \pm 1.0$ & $3.7 \pm 1.0$ & $3.9 \pm 1.4$ & $0.06(-0.07 ; 0.19)$ & 0.38 \\
\hline VLDL-cholesterol & $0.7 \pm 0.4$ & $0.7 \pm 0.4$ & $0.8 \pm 0.4$ & $0.03(-0.02 ; 0.08)$ & 0.21 \\
\hline Serum triglyceride & $1.4(0.9-2.0)$ & $1.4(1.1-2.0)$ & $1.5(1.1-2.6)$ & $5.2 \%(0.4 \% ; 10.0 \%)$ & 0.03 \\
\hline
\end{tabular}

Data are unadjusted means \pm SD or medians (interquartile range). Values of serum triglycerides were logarithmically transformed before statistical analysis, and their effect sizes are presented as the increase/decrease in percent. Normoglycemic: glucose-tolerant individuals from the Inter99 cohort. Hyperglycemic: individuals with IFG, IGT or screen-detected T2D from the Inter99 cohort. All analyses were made using an additive genetic model, adjusting for age, sex, and BMI. ACADS rs2014355 genotypes: WT, homozygous major T-allele carriers; HE, heterozygous; HO, homozygous minor C-allele carriers.

carrying the $A C A D S$ rs2014355 minor C-allele we found significantly decreased glucose stimulated insulin release during an OGTT measured as decreased serum insulin levels at $30 \mathrm{~min}$, decreased incremental AUC for serum insulin, decreased BIGTT-AIR, decreased AUC for insulin/AUC for glucose ratio, as well as significantly increased

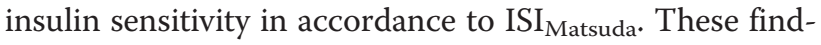
ings were reproduced, although to a lower significance level when all treatment-naïve individuals from the Inter99 were examined. In contrast, rs11161510 of $A C A D M$ did not associate with any of the examined quantitative traits or with diabetes in case-control analysis.

We did not find any consistent associations of rs2014355 of ACADS with estimates of insulin sensitivity; thus the C-allele did not associate with BIGTT-S $\mathrm{S}_{\mathrm{i}}$ or HOMA-IR but only with ISI $_{\text {Matsuda. We have no obvious }}$ explanation for this inconsistency. Yet, the $\mathrm{C}$-allele also failed to associate with a HOMA-derived disposition index, which is a measure of $\beta$-cell function and therefore we cannot exclude that individuals homozygous for the minor $\mathrm{C}$-allele show decreased insulin release as an adaptive response to a primarily improved insulin sensitivity within this genotype group. This interpretation of data is in line with the other insulin sensitivity indices (HOMA-IR and BIGTT- $\mathrm{S}_{\mathrm{i}}$ ) that also show a tendency to higher estimates of insulin sensitivity among $\mathrm{C}$-allele carriers, although not statistically significant.
Both T2D and disorders related to ACADS show large heterogeneity in the clinical setting which challenges the search for a clear relationship between the two. Additionally, the large variations in phenotypic traits associated with multiple defects of $A C A D S$ may imply that specific polymorphisms only have a mild regulatory effect. As Gieger et al. report, these variants are not associated with severe physiological abnormalities but instead a moderate phenotypic expression may be common in the population. Furthermore, dehydrogenases of other FA's of varying chain-length may partially compensate for the polymorphism and thereby hide the effect or manifest an altered phenotype. Consequently, other interrelating factors, either of environmental or genetic origin, may be necessary to predispose ACADS rs2014355 risk allele carriers to $\beta$-cell dysfunction.

Under fasting states or other stressing conditions such as fever, defects of $A C A D S$ have been linked to hypoglycemia $[14,26,27]$. These findings may be explained by decreased hepatic gluconeogenesis due to FA accumulation and increased utilization of glucose as a fuel because the defective dehydrogenase will prevent the SCFA to be oxidized. However in non-fasting states when there are abundant sources of carbohydrates and fats available, it could be hypothesized, that polymorphisms in ACADS would lead to accumulation of SCFA which instead will be converted to complex lipid signaling 
molecules and thereby synergize with the glucose to stimulate insulin secretion leading to hyperinsulinemia. In the chronic state this could potentially desensitize the insulin receptors, down-regulate insulin release and finally exhaust the $\beta$-cell leading to reduced $\beta$-cell function.

Together with the additional effect of the increased circulating and tissue FAs, which due to lipotoxicity may operate to diminish the relatively increased insulin sensitivity, a reduced $\beta$-cell function could possibly predispose some individuals to T2D. However, the ACADS variant did not associate with increased risk of developing T2D in the present case-control study, which is in concordance with the DIAGRAM meta-analysis (stage 1: 4,549 T2D cases and 5,579 control individuals), where rs2014355 shows no association with T2D (OR 1.02 (0.94-1.12), $P_{\text {additive }}=0.6$, under the random effects model) [28]. In our study, we are only able to exclude with $80 \%$ likelihood that the OR is $>1.10$. This point to the fact, that none of the two mentioned studies have sufficient statistical power to detect smaller effects of the variant on the risk of developing T2D. It is recognized that many case-control studies of multifactorial diseases with a complex mode of inheritance, e.g., T2D, often are statistically underpowered in yielding conclusive results, and random spurious positive associations due to multiple testing often cannot be excluded. None of the results presented in this paper can withstand correction for multiple testing. Therefore, whether the associations between the $A C A D S$ variant and examined phenotypes are chance findings, need further investigation in larger well-powered study samples.

\section{Conclusion}

We provide suggestive evidence that the C-allele of rs2014355 of ACADS associates with reduced measures of glucose-stimulated insulin release during an OGTT, indicating that the link to T2D previously suggested for this variant, may be mediated through an impaired $\beta$-oxidation of fatty acids [10]. However, in the present study we found no evidence of an increased risk of T2D for the C-allele of rs2014355 of ACADS. rs11161510 of $A C A D M$ did not associate with any of the examined T2D related traits. Further investigation is needed before conclusive remarks can be appointed to the variants in $A C A D S$ and $A C A D M$ and their putative involvement in the pathogenesis of diabetes.

\section{Additional material}

Additional file 1: Characteristics for individuals included in the study stratified according to study group. A table showing the number of individuals included from each study group and their characteristics (as unadjusted means \pm SD)
Additional file 2: Anthropometric characteristics of successfully genotyped Danes from the Inter99 cohort. A table showing the anthropometric characteristics (as unadjusted means \pm SD) of successfully genotyped glucose-tolerant Danes from the Interg9 cohort stratified according to ACADS and ACADM genotypes, including effect sizes and corresponding p-values

\section{List of abbreviations}

AIR: acute insulin response; BIGTT-AIR: BIGTT-acute insulin response; BIGTT-S: BIGTT-insulin sensitivity index; Cl: confidence interval; CPT-I: carnitinepalmitoyl-transferase I; FA: fatty acids; FFA: free fatty acids; GSIS: glucosestimulated insulin secretion; GWAS: genome-wide association study; IGT: impaired glucose tolerance; ISI: insulin sensitivity index; MAF: minor allele frequency; MCAD: medium-chain Coenzyme A dehydrogenase; NAFLD: nonalcoholic fatty liver disease; NGT: normal glucose tolerance; OGTT: oral glucose tolerance test; OR: odds ratio; SCAD: short-chain Coenzyme-A dehydrogenase; SCFA: short-chain fatty acids; TG: triglyceride; T2D: type 2 diabetes.

\section{Acknowledgements}

The authors wish to thank A. Forman, I.-L. Wantzin, and M. Stendal for technical assistance, and A. L. Nielsen, G. Lademann, and M.M.H. Kristensen for management assistance. The study was supported by grants from: the Lundbeck Foundation Centre of Applied Medical Genomics for Personalized Disease Prediction, Prevention and Care (LuCAMP), the Danish Health Research Council, "Hepatic and adipose tissue and functions in the metabolic syndrome" (HEPADIP http://www.hepadip.org), which is supported by the European Commission as an integrated project under the 6th Framework Programme (LSHM-CT-2005-018734), the Danish Diabetes Association, the Danish Council for Independent Research (Medical Sciences) and Novo Nordisk. The Inter99 was initiated by T. Jørgensen (Principal Investigator), K. Borch-Johnsen (co-Principal Investigator), H. Ibsen and T. F. Thomsen. The steering committee comprises the former two and C. Pisinger. The study was financially supported by research grants from the Danish Research Council, the Danish Centre for Health Technology Assessment, Novo Nordisk, Research Foundation of Copenhagen County, Ministry of Internal Affaires and Health, the Danish Heart Foundation, the Danish Pharmaceutical Association, the Augustinus Foundation, the Ib Henriksen Foundation, the Becket Foundation and the Danish Diabetes Association. The Marie Krogh Center for Metabolic Research is funded by the Novo Nordisk Foundation.

\section{Author details}

'Marie Krogh Center for Metabolic Research, Section of Metabolic Genetics, Faculty of Health Sciences, University of Copenhagen, Denmark. ${ }^{2}$ Faculty of Pharmaceutical Sciences, University of Copenhagen, Copenhagen, Denmark. ${ }^{3}$ Hagedorn Research Institute, Gentofte, Denmark. ${ }^{4}$ Institute of Biomedical Science, Faculty of Health Sciences, University of Copenhagen, Copenhagen, Denmark. ${ }^{5}$ Novo Nordisk A/S, Medical and Science, Development Projects, Bagsværd, Denmark. ${ }^{6}$ Department of General Practice, University of Aarhus, Aarhus, Denmark. ${ }^{7}$ Research Centre for Prevention and Health, Glostrup University Hospital, Glostrup, Denmark. ${ }^{8}$ Steno Diabetes Center, Gentofte, Denmark. Institute of Preventive Medicine, Copenhagen University Hospital, Center for Health and Society, Copenhagen, Denmark. ${ }^{10}$ Faculty of Health Sciences, University of Aarhus, Aarhus, Denmark. ${ }^{11}$ Faculty of Health Sciences, University of Southern Denmark, Odense, Denmark.

\section{Authors' contributions}

The concept and idea regarding the epidemiological studies underlying the study populations was conceived by $\mathrm{CP}, \mathrm{AS}, \mathrm{TL}, \mathrm{OP}$, and TH. The collection of study subjects was planned and performed by $C P, A S, T L, O P$, and $T H$. The original hypothesis regarding the study was conceived by $\mathrm{KB}$ and $\mathrm{MH}$ and approved by OP and TS, TH. Detail planning of analyses and study design was performed by $\mathrm{KB}$ and $\mathrm{MH}$ and approved by $\mathrm{OP}$ and $\mathrm{TH}$. $\mathrm{KB}, \mathrm{MH}$, $\mathrm{JJ}, \mathrm{DW}, \mathrm{OP}$, and TH contributed to the establishment of study population databases specific for this study. Statistical analyses in association studies were performed by $\mathrm{KB}, \mathrm{MH}$ and JJ. NK, CS, and $\AA \mathrm{A}$ helped to draft the 
manuscript. The first manuscript was written by $\mathrm{MH}, \mathrm{KB}$, and $\mathrm{J}$ with equal contribution and the final draft was finalized by $K B, T S, O P$ and $T H$. All authors revised the manuscript and contributed to the discussion. The final manuscript was read and approved by all authors.

\section{Competing interests}

K. Banasik, C.H. Sandholt, T. Hansen and O. Pedersen hold employee shares in Novo Nordisk A/S. All other authors declare that there is no competing interest associated with this manuscript.

Received: 2 June 2010 Accepted: 6 January 2011

Published: 6 January 2011

\section{References}

1. Newsholme P, Keane D, Welters HJ, Morgan NG: Life and death decisions of the pancreatic beta-cell: the role of fatty acids. ClinSci(Lond) 2007, 112(1):27-42.

2. Schaffer JE: Lipotoxicity: when tissues overeat. CurrOpinLipidol 2003, 14(3):281-287.

3. Kelley DE, Goodpaster B, Wing RR, Simoneau JA: Skeletal muscle fatty acid metabolism in association with insulin resistance, obesity, and weight loss. AmJPhysiol 1999, 277(6 Pt 1):E1130-E1141.

4. Zhang $Y$, Xiao M, Niu G, Tan H: Mechanisms of oleic acid deterioration in insulin secretion: role in the pathogenesis of type 2 diabetes. Life Sci 2005, 77(17):2071-2081.

5. Biden TJ, Robinson D, Cordery D, Hughes WE, Busch AK: Chronic effects of fatty acids on pancreatic beta-cell function: new insights from functional genomics. Diabetes 2004, 53(Suppl 1):S159-S165.

6. Kelley DE, Mandarino $\mathrm{L}$ : Fuel selection in human skeletal muscle in insulin resistance: a reexamination. Diabetes 2000, 49(5):677-683.

7. Haber EP, Ximenes HM, Procopio J, Carvalho CR, Curi R, Carpinelli AR: Pleiotropic effects of fatty acids on pancreatic beta-cells. JCell Physiol 2003, 194(1):1-12.

8. Nolan CJ, Madiraju MS, ghingaro-Augusto V, Peyot ML, Prentki M: Fatty acid signaling in the beta-cell and insulin secretion. Diabetes 2006, 55(Suppl 2):S16-S23.

9. Keane D, Newsholme P: Saturated and unsaturated (including arachidonic acid) non-esterified fatty acid modulation of insulin secretion from pancreatic beta-cells. BiochemSocTrans 2008, 36(Pt 5):955-958.

10. Gieger C, Geistlinger L, Altmaier E, Hrabe de AM, Kronenberg F, Meitinger T, Mewes HW, Wichmann HE, Weinberger KM, Adamski J, Illig T, Suhre K: Genetics meets metabolomics: a genome-wide association study of metabolite profiles in human serum. PLoSGenet 2008, 4(11):e1000282.

11. Eaton S, Chatziandreou I, Krywawych S, Pen S, Clayton PT, Hussain K: Shortchain 3-hydroxyacyl-CoA dehydrogenase deficiency associated with hyperinsulinism: a novel glucose-fatty acid cycle? BiochemSocTrans 2003, 31(Pt 6):1137-1139.

12. Haber EP, Procopio J, Carvalho CR, Carpinelli AR, Newsholme P, Curi R: New insights into fatty acid modulation of pancreatic beta-cell function. IntRevCytol 2006, 248:1-41.

13. Gregersen N, Andresen BS, Corydon MJ, Corydon TJ, Olsen RK, Bolund L, Bross P: Mutation analysis in mitochondrial fatty acid oxidation defects: Exemplified by acyl-CoA dehydrogenase deficiencies, with special focus on genotype-phenotype relationship. HumMutat 2001, 18(3):169-189.

14. Pedersen CB, Kolvraa S, Kolvraa A, Stenbroen V, Kjeldsen M, Ensenauer R, Tein I, Matern D, Rinaldo P, Vianey-Saban C, Ribes A, Lehnert W, Christensen E, Corydon TJ, Andresen BS, Vang S, Bolund L, Vockley J, Bross $P$, Gregersen N: The ACADS gene variation spectrum in 114 patients with short-chain acyl-CoA dehydrogenase (SCAD) deficiency is dominated by missense variations leading to protein misfolding at the cellular level. HumGenet 2008, 124(1):43-56.

15. Clayton PT, Eaton S, ynsley-Green A, Edginton M, Hussain K, Krywawych S, Datta V, Malingre HE, Berger R, van dBl: Hyperinsulinism in short-chain L3-hydroxyacyl-CoA dehydrogenase deficiency reveals the importance of beta-oxidation in insulin secretion. JClin/nvest 2001, 108(3):457-465.

16. Herrema H, Derks TG, van Dijk TH, Bloks WW, Gerding A, Havinga R, Tietge UJ, Muller M, Smit GP, Kuipers F, Reijngoud DJ: Disturbed hepatic carbohydrate management during high metabolic demand in mediumchain acyl-CoA dehydrogenase (MCAD)-deficient mice. Hepatology 2008, 47(6):1894-1904.
17. Tolwani RJ, Hamm DA, Tian L, Sharer JD, Vockley J, Rinaldo P, Matern D, Schoeb TR, Wood PA: Medium-chain acyl-CoA dehydrogenase deficiency in gene-targeted mice. PLoSGenet 2005, 1(2):e23.

18. Wood PA, Amendt BA, Rhead WJ, Millington DS, Inoue F, Armstrong D: Short-chain acyl-coenzyme A dehydrogenase deficiency in mice. PediatrRes 1989, 25(1):38-43.

19. Schuler AM, Wood PA: Mouse models for disorders of mitochondrial fatty acid beta-oxidation. ILARJ 2002, 43(2):57-65.

20. Lauritzen T, Griffin S, Borch-Johnsen K, Wareham NJ, Wolffenbuttel BH, Rutten G: The ADDITION study: proposed trial of the cost-effectiveness of an intensive multifactorial intervention on morbidity and mortality among people with Type 2 diabetes detected by screening. IntJObesRelat Metab Disord 2000, 24(Suppl 3):S6-11.

21. Jørgensen T, Borch-Johnsen K, Thomsen TF, Ibsen H, Glümer C, Pisinger C: A randomized non-pharmacological intervention study for prevention of ischaemic heart disease: baseline results Inter99. EurJCardiovascPrevRehabil 2003, 10(5):377-386.

22. World Health Organization Study G: Definition, Diagnosis and Classification of Diabetes Mellitus and its Complications. Part 1: Diagnosis and Classification of Diabetes Mellitus. Tech Rep Ser WHO/NCD/ NCS/992 Geneva: World Health Organization; 1999.

23. Matthews DR, Hosker JP, Rudenski AS, Naylor BA, Treacher DF, Turner RC: Homeostasis model assessment: insulin resistance and beta-cell function from fasting plasma glucose and insulin concentrations in man. Diabetologia 1985, 28(7):412-419.

24. Hansen T, Drivsholm T, Urhammer SA, Palacios RT, Volund A, BorchJohnsen K, Pedersen O: The BIGTT test: a novel test for simultaneous measurement of pancreatic beta-cell function, insulin sensitivity, and glucose tolerance. Diabetes Care 2007, 30(2):257-262.

25. Matsuda M, DeFronzo RA: Insulin sensitivity indices obtained from oral glucose tolerance testing: comparison with the euglycemic insulin clamp. Diabetes Care 1999, 22(9):1462-1470.

26. van Maldegem BT, Duran M, Wanders RJ, Niezen-Koning KE, Hogeveen $M$, lj|st L, Waterham HR, Wijburg FA: Clinical, biochemical, and genetic heterogeneity in short-chain acyl-coenzyme A dehydrogenase deficiency. JAMA 2006, 296(8):943-952.

27. Rinaldo P, Matern D, Bennett MJ: Fatty acid oxidation disorders. AnnuRevPhysiol 2002, 64:477-502.

28. Zeggini E, Scott LJ, Saxena R, Voight BF, Marchini JL, Hu T, de Bakker PI, Abecasis GR, Almgren P, Andersen G, Ardlie K, Bostrom KB, Bergman RN, Bonnycastle LL, Borch-Johnsen K, Burtt NP, Chen H, Chines PS, Daly MJ, Deodhar P, Ding CJ, Doney AS, Duren WL, Elliott KS, Erdos MR, Frayling TM, Freathy RM, Gianniny L, Grallert H, Grarup N, Groves CJ, Guiducci C, Hansen T, Herder C, Hitman GA, Hughes TE, Isomaa B, Jackson AU, Jorgensen T, Kong A, Kubalanza K, Kuruvilla FG, Kuusisto J, Langenberg C, Lango H, Lauritzen T, Li Y, Lindgren CM, Lyssenko V, Marvelle AF, Meisinger C, Midthjell K, Mohlke KL, Morken MA, Morris AD, Narisu N, Nilsson P, Owen KR, Palmer CN, Payne F, Perry JR, Pettersen E, Platou C, Prokopenko I, Qi L, Qin L, Rayner NW, Rees M, Roix JJ, Sandbaek A, Shields B, Sjogren M, Steinthorsdottir V, Stringham HM, Swift AJ, Thorleifsson G, Thorsteinsdottir U, Timpson NJ, Tuomi T, Tuomilehto J, Walker M, Watanabe RM, Weedon MN, Willer CJ, Illig T, Hveem K, Hu FB, Laakso M, Stefansson K, Pedersen O, Wareham NJ, Barroso I, Hattersley AT, Collins FS, Groop L, McCarthy MI, Boehnke M, Altshuler D: Meta-analysis of genome-wide association data and large-scale replication identifies additional susceptibility loci for type 2 diabetes. Nat Genet 2008, 40(5):638-645.

\section{Pre-publication history}

The pre-publication history for this paper can be accessed here: http://www.biomedcentral.com/1471-2350/12/4/prepub

doi:10.1186/1471-2350-12-4

Cite this article as: Hornbak et al:: The minor C-allele of rs2014355 in $A C A D S$ is associated with reduced insulin release following an oral glucose load. BMC Medical Genetics 2011 12:4. 